\title{
Distinguishing Pattern-Types in Printed Documents
}

\author{
Georg Lokowandt, Waltraud Schweikhardt \\ Institut für Informatik \\ Universität Stuttgart, Germany
}

\begin{abstract}
Reading machines and other devices have helped a lot in integrating blind persons into society. Simultaneously the progress and widespread use of desktop-publishing systems lead to documents that are hard to recognise for current reading machines. Nevertheless, access to printed documents is required by most blind persons. In this article we show a possible structure of an improved and flexible system for document recognition. It will be capable of processing any printed document, for instance a letter or a musical score as well as diagrams or line drawings. Special emphasis is put on correct and efficient distinction of categories of documents. Requirements for distinction are shown and an suggestion for an implementation is presented. It will perform this classification in a fast and flexible way. Also, the suggested method can automatically as well as interactively classify the type of a document. Afterwards the contents of the document can be displayed in an appropriate style. The blind users also have the possibility to interactively define new classes of documents if required. The prototype of this system is currently being implemented at our institute.
\end{abstract}

\section{Motivation}

To read the contents of a printed document without the help of a sighted person, blind people can fall back on computerised equipment. For most computers, there are OCR (Optical Character Recognition)-systems available, that provide the possibility to read out recognised text. But many documents can not be processed to be adequately represented for the blind. Especially documents, that are not composed of a character set, like graphics and diagrams, can not be processed by today's OCRsystems. In these cases the content of the document has to be read with the Optacon, copied on swelling paper or analysed with similar devices. As shown in Figure 1, ways to process an unknown document, which are available today (OCR, Optacon and swelling paper) share the problem, that non of these devices is capable of processing every content of a document. If help by a sighted person is not available or not wanted because of privacy considerations, the document analysis is a very time-consuming process.

Our goal is to develop a system to support the blind in reading and exploring any printed document on their own. The program should appear like an expanded OCRSystem. Graphics shall be represented in a reasonable way. 


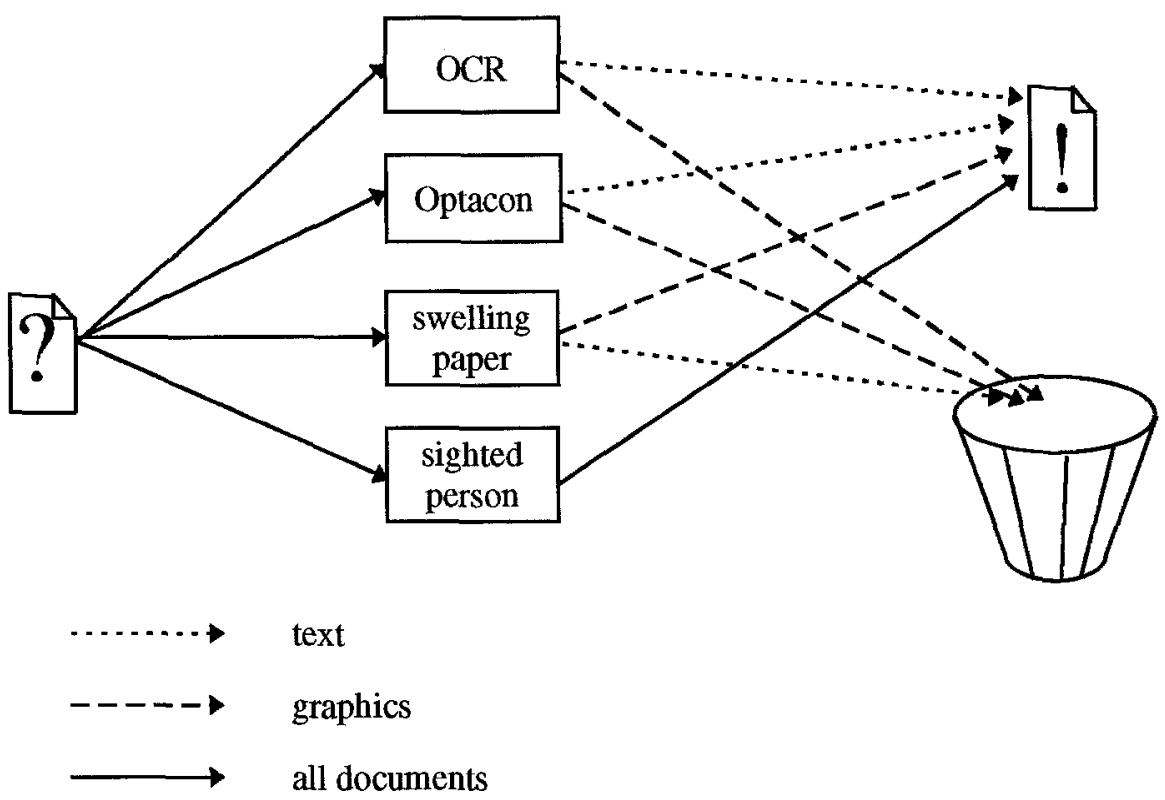

Figure 1: Current Ways of Understanding the Contents of a Printed Document

\section{Basics}

Experiments have shown that many documents which include graphics can be understood without big difficulty if they are presented in the proper magnitude or if only some aspects are shown at a time. It is difficult, but not impossible for blind persons to determine a suitable magnitude on their own. If the document shows an unknown item in an unknown format the examination will take several iterations. First the right face of the page and the correct orientation have to be determined. Afterwards, most of the participants of our tests tried to find text passages and read them, in order to conclude the contents of the whole document from what they read. By understanding the text, most people were able to relate the document to a specific topic. If the tested persons were familiar with this topic, they also knew of some classes of documents that are usually used to present this information. By putting the document in one of these categories, it is easy to decide whether to proceed with the analysis. Also the decision what tools are to be used to process this kind of document is made after the classification. For example in a document showing a musical score it is searched for other tokens than in a document showing a pie chart or a diagram. While the tactile copy of a diagram can be used to get the required overview, the position of each note is essential for reading a score. Therefore an Optacon is the 
better choice for getting the details of a score. To select the right tool for further processing and therefore to understand the contents of the printed document requires a correct classification of the document.

A blind user will classify a document according to its semantic characteristics. A program, however, does not have this ability. On the other hand, many syntactic features of a printed document can easily be determined. The existence of lines and their arrangement, the usage of colour or the usage of text that is emphasised by changing its background colour, can be determined by well-known algorithms. Classification of this kind resembles to the classification performed by a sighted person. A sighted person is able to recognise the use of colours as well as the use of lines and coloured areas at a glance. Experience helps to classify a document according to its visual syntactic features before reading it. We therefore believe, that syntactic features are sufficient for classification of printed documents.

\section{Reflections on Current Recognition Systems}

It has been suggested to use neural nets or fuzzy-logic controllers to perform the classification. Also rule-based systems are applied to the problem of document classification and document analysis. Usually the whole process of document recognition is divided into two phases, the feature extraction and the contents recognition. In the first phase syntactic features are extracted and in the second one, the contents of the document are extracted by application of neural nets or fuzzy controllers. Without a separate phase of document classification these approaches are only capable of processing a small number of document classes. Usual OCR-Systems can only process text and another program has to be used to process for instance musical scores. The classification phase is implicitly performed by the user who has to know which program to use. There is another disadvantage that follows from feature extraction being a separate step. A fixed set of routines for feature extraction has to be used for every document. Afterwards, a fast and flexible method is used for recognition. The less features are extracted, the less time is required but the less documents can be recognised correctly. The number of features extracted therefore depends on the time that might be spend during feature extraction and the accuracy required for the recognition process. If this approach is generalised on arbitrary printed documents, the number of required algorithms for feature extraction and therefore the recognition time, rises. An additional disadvantage of this approach depends on the requirement, that every algorithm must compute useful results for every document. When the extraction algorithms are generalised to process a wider variety of document classes, they usually get more complicated and require more computation time.

\section{Suggested Solution}

Our approach suggests to merge the phase of feature extraction with the new phase of document classification. Whenever a feature of the document is extracted, the document should be classified according to this feature. When the class of the 
document is determined with an acceptable amount of certainty this phase should be ended and the recognition phase should begin.

To allow a program to perform the classification, three groups of routines have to be implemented:

- routines to extract relevant information,

- routines to administer document classes,

- routines to control the classification.

When the computation of all relevant syntactic features can be expressed by algorithms, an important step towards automatic classification will be done.

Another step has to be the definition of appropriate document classes. This goal, however, will never be achieved completely, because new document classes can and will be developed at all times. Nevertheless, there is a small number of general document classes which are sufficient for processing most of the documents. Obviously, these basic classes must be provided from the beginning. To work quickly and efficiently, additional document classes can be defined on demand. In order to do this, the system must be open to new document classes. The blind users should be able to perform these additions on their own.

This concept of an expandable class structure and a few general classes helps to integrate the intelligence of the user to recognise and perform necessary additions. Therefore our program itself does not need to integrate components for acquiring knowledge and ends up in a less complex result.

The last part of the classification system are the routines that control the classification. They use the extracted features to assign a given document to a defined class of documents. This is a typical problem of decision finding which is not easy but not unsolvable. Attention has to be paid that the whole classification is performed within a reasonable time for interactive work.

\section{Terms}

\section{Document Classes}

A class of documents is a set of documents that share a set of common features. Each document is an instance of a hierarchy of document-classes, but only the most specialised class is called the class to which the document belongs or the type of the document. For each class there is an arrangement of specialised routines to extract the contents of documents of one kind. Also there are routines to represent the information to the user. Examples of classes of documents are:

- raw text

- business forms

- mathematical formulas

- line drawings

- music charts

- composed documents. 
This enumeration can easily be continued to any length. Some users might want to distinguish between a business letter and a private letter, while other users might want to use the document class petri-net.

\section{Explicit Information}

This kind of information consists of all characters, symbols, graphical lines etc. with all their attributes as size, font etc. Also the layout of the document may be included. It resembles to syntactic information. This information is extracted in the analysisphase.

\section{Implicit Information}

This term stands for all kinds of information that are extracted during the classification process. We could also call it meta-information because this information describes the class of a document and not its contents. For example, a text containing formulas has a mathematical content, although this meaning needn't be expressed anywhere in the printed text.

\section{Implementation}

The whole process of document recognition is divided into several phases. These phases are described in the following sections.

\section{Document Classification}

Before the explicit information within the document can be extracted, the class the document belongs to has to be determined.

The routine to solve this task uses an expandable decision tree, as shown in Figure 2 and initialises a list of attributes, that characterise the document after its way through the decision tree. The algorithm controlling the classification passes every document trough this tree. At every node it is decided which branch has to be taken. Each node within the tree contains the algorithm which has to be applied next and the branch which has to be taken after the algorithm has extracted another syntactic feature of the document. This feature is added to the list of attributes.

When a leaf is reached, the classification phase is completed and the type of the document is determined. If the document, however, does not belong to any of the known types, all implicit information that has been extracted, is presented to the user. Since the classification would end without a result, the user must be able to interfere into the classification process. 


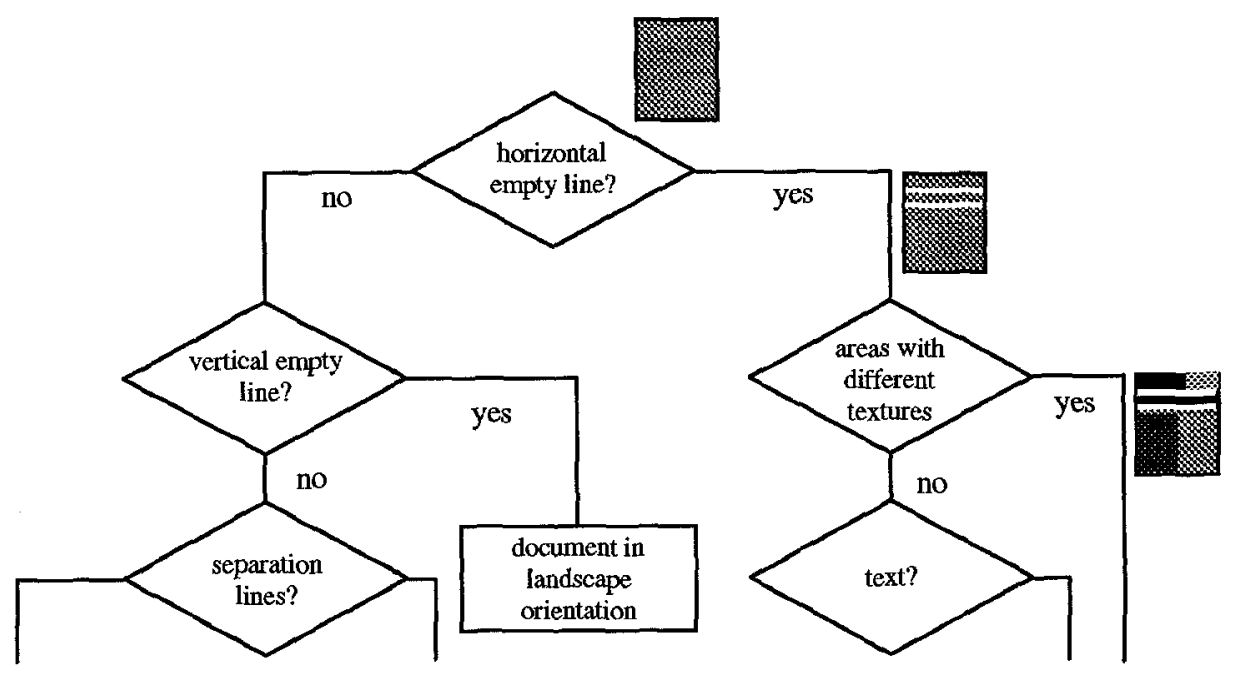

Figure 2: Decision Tree for the Document Classification (part)

Often a document is composed of several areas with different patterns. In these cases the analysing algorithm can divide the document. Afterwards, the analysing algorithm can call the classification routine again for every area. The decision tree in Figure 2 shows a leaf to handle a document in landscape-format. In the analysisphase, the contents of the document will be turned to portrait-format and afterwards be processed as any new document.

\section{Phase of Analysing}

During the phase of analysing, the information, which is comprised in the document is represented in an internal data-structure adequate to the document type. Plain text is represented by ASCII-Characters, control characters for font, sizes and other attributes. All text attributes have to be recognised and stored within the internal data structure as well as the layout of the printed text. This information is required as a whole in order to enable the blind and sighted to work together. In general, the internal data-structure should be suited to hold all information that is required to work with a document of the specific class. In addition all information that is required to cooperate with a sighted person should be stored. The analysis is performed by a set of special routines which call a library of general image processing routines.

\section{Representation of Results}

When all information that is included in the document, is extracted and converted into the internal data-structure, the representation of this information is started. For 
each class of documents, there is a special routine to control the presentation. All these routines can use a library of general dialogue-routines. This library manages a set of input and output devices. Output devices we use primarily are a Brailledisplay, a speech output device, a pin matrix device and a text- as well as a Windows screen. According to the used output device, we want to use the cursor routing keys, the keyboard, the pin matrix device or a mouse for the input. Several of these devices should be used simultaneously or on their own. In order to store the results of processing a printer and files must also be supported. For the analysis only devices which can be used interactively should be used.

\section{Building up the Decision Tree}

The classification of documents as described above requires a decision tree that holds the information on how to perform the classification. During the usual classification the decision tree is unchanged. Whenever a new class of documents is defined and whenever a new algorithm for the extraction of syntactic features is implemented, the tree has to be rebuild. The time that is spend during this process doesn't matter because it is performed seldom and doesn't require any user interaction. Therefore we can apply all algorithms for extracting syntactic features on every available document. Afterwards a clustering-algorithm calculates the significance of every syntactic feature for the distinction between document classes. The quotient of significance and runtime of an algorithm determines its position within the decision tree. The fastest algorithm with the most significant results is put at the root of the tree while the slow algorithms are positioned near the leafs.

\section{Summary}

In this paper, we introduce our strategy to split the process of recognising a printed document into two phases. We always start with a classification phase which is followed by an analysis phase, if the document's type has been determined. Since we also consider composed documents, it is possible that a further phase of classification will be necessary for a part of the document later on. We believe that our system will finally process almost any printed document in an adequate manner. By storing and presenting explicit as well as implicit information, the user gets the possibility to understand and influence the recognition process in case of errors. The described scheme requires and promotes interactive work.

\section{Literature}

Nadine Baptiste, Monique Truquet

A Complete Solution to Help a Blind Musician to Access Musical Data

Proceedings of the ECART 2 Conference, Stockholm, Sweden, May 26-28, 1993

Inhao Chang, Murray Loew

Pattern Recognition with New Class Discovery

Proceedings of the CVPR Conference, June 3-6, 1991 pp. 438-443 
Alan Y. Commike

Syntactic Pattern Classification by Branch and Bound Search

Proceedings of the CVPR Conference, June 3-6, 1991 pp. 432-437

Andreas Dengel

Automatische Visuelle Klassifikation von Dokumenten

Ph. D. Dissertation an der Fakultät Informatik, Universität Stuttgart, 1989

Gaby Glanzman, Bernhard Arnolds and Uwe Koch

Evaluation of Text Scanners (Reading Machines) available for general use at the

University Library, Freiburg

Proceedings of the ECART 2 Conference, Stockholm, Sweden, May 26-28, 1993

Wolfgang A. Kreissl

Interaktives Erkunden gedrucker Dokumente

Diploma thesis Nr. 794, Institut für Informatik, Univ. Stuttgart, 1991

Urs Mueller, Markus Schenkel

Improving Reading Machines for the Blind with Interactive Document Segmentation

Computers for Handicapped Persons

Proceedings of the 3rd International Conference, Vienna, July 7-9, 1992 pp. 363-372

Martin Recker

Erstellen einer tastbaren Orientierungsseite zu gedruckten Dokumenten

Diploma thesis Nr. 717, Institut für Informatik, Univ. Stuttgart, 1990

Waltraud Schweikhardt

Interaktives Exkunden tastbarer Grafiken durch Blinde

Bullinger, H.-J. (Hrsg.), Software-Ergonomie '85,

B.G. Teubner Stuttgart, 1985, pp. 366-375.

Waltraud Schweikhardt

Bildschirmtext - ein Rechnerunterstïtztes Kommunikationsmittel auch für Blinde

Hansen, H. R. (Hrsg.), GI/OCG/ÖGI Jahrestagung 1985

Springer-Verlag 1985, pp. 691-701.

Waltraud Schweikhardt

From Printed Media to Tactile Documents

Workshop „New Problems and Future Solutions for Man Machine Interaction“

Stuttgart, Dec. 16-17, 1991

Wolfgang L. Zagler, Johann Haider, Peter Mayer, Franz Peter Seiler and Michael Busboom Technology for Supporting Mainstream-Education

Proceedings of the ECART 2 Conference, Stockholm, Sweden, May 26-28, 1993 